\title{
Research Paper: Differential Expression of CXCL1, CXCL10, and CXCL12 in Response to Cerebral Ischemic Postconditioning in Rat Brain
}

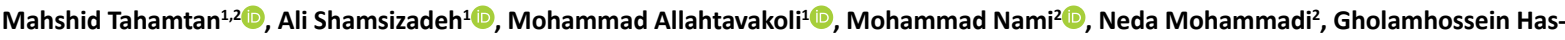
sanshahi $^{3^{*}}(\mathbb{D})$

1. Physiology Pharmacology Research Center, Rafsanjan University of Medical Sciences, Rafsanjan, Iran.

2. Department of Neuroscience, School of Advanced Medical Sciences and Technologies, Shiraz University of Medical Sciences, Shiraz, Iran.

3. Molecular Medicine Research Center, Rafsanjan University of Medical Sciences, Rafsanjan, Iran.

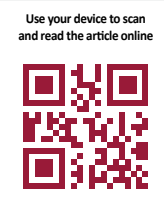

Citation: Tahamtan M, Shamsizadeh A, Allahtavakoli M, Nami M, Mohammadi N, Hassanshahi Gh. Differential Expression of CXCL1, CXCL10, and CXCL12 in Response to Cerebral Ischemic Postconditioning in Rat Brain. JAMSAT. 2017; 3(4):197-204. https://doi.org/10.32598/jamsat.3.4.197

doij https://doi.org/10.32598/jamsat.3.4.197

Article info:

Received: 17 Apr 2017

Accepted: 09 Sep 2017

Keywords:

Chemokine, Global cerebral ischemia, Postconditioning

\section{ABSTRACT}

Objectives: Cerebral ischemic preconditioning lessens stroke-induced injuries, but it is clinically feasible only when the occurrence of stroke is predictable. Reperfusion plays a critical role against cerebral injury after stroke; we tested the hypothesis that interrupting ischemia during early reperfusion, i.e. Postconditioning (POCO) affects CXC chemokine expression and further reduce inflammation in rat model of ischemia/reperfusion.

Materials \& Methods: Adult male Wistar rats (250-300 g) were used in this experiment. Using 4-vessel occlusion method, global cerebral ischemia was induced and POCO was performed by applying 3 cycles of 15 -s/15-s reperfusion/reocclusion after a 45 -s reperfusion (POCO45-15/15). Western blotting analysis was used to investigate CXCL1, CXCL10 and CXCL12 expression $24 \mathrm{~h}, 48 \mathrm{~h}$ and one week after ischemic postconditioning (iPOCO).

Results: Based on the results, iPOCO attenuates the expression of inflammatory chemokines CXCL1 and CXCL10 in hippocampus area of postconditioned rats, while the CXCL12 was not affected by iPOCO.

Conclusion: Current findings may support chemokines role in $\mathrm{PPOCO}$ via reduction of inflammation. Also there could be a link between postconditioning, stress and inflammation through chemokines.

\section{Introduction}

erebrovascular accidents are amongst the leading causes of either death or permanent disability without an effec- tive treatment so far [1]. Extensive research has been aimed at finding effective strategies to ameliorate Ischemia/Reperfusion (I/R) injury. Research in treating this type of injury has led to the discovery of the two recently introduced concepts; preconditioning

* Corresponding Author:

Gholamhossein Hassanshahi, MD

Address: Molecular Medicine Research Center, Rafsanjan University of Medical Sciences, Rafsanjan, Iran.

Tel: +98 (939) 15234003

E-mail: ghhassanshahi@rums.ac.ir 
and postconditioning. Preconditioning has been demonstrated to seriously protect against I/R injury. However, its clinical application is only possible when the occurrence of stroke is predictable and controllable.

Immediate initiation of reperfusion is the most efficacious treatment to reduce I/R injury. However, reperfusion may cause additional injury, too. Another newly established concept that lacks the clinical restrictions of preconditioning is postconditioning in which brief repetitive cycles of ischemia with intermittent reperfusion is followed by prolonged ischemia elicited tissue protection [2, 3].

The protective role of postconditioning is well demonstrated in brains of different animal models such as rats [4-6], rabbits [7], and mice [8]. Nevertheless, whether postconditioning has protective effects against inflammation induced by global cerebral I/R injury is yet to be fully investigated. Experimental studies have indicated that infiltration of inflammatory cells, including neutrophils, tissue mast cells, monocytes and platelets during reperfusion have a crucial role in I/R injury. These cells contribute to the phenomenon of I/R by releasing oxygen-derived free radicals, proteases and leukotrienes.

Thus, modulation of cytokine induced inflammatory responses may have a direct impact on I/R injury and free flap survival [9]. Chemokines are low molecular weight proteins (8-17 kDa) [10]. A growing body of evidence has indicated the involvement of chemokines under pathological and repair circumstances in CNS [11, 12]. Thus our research team aimed to examine whether chemokines play a role in postconditioning and hence reduce inflammation in rat model of $\mathrm{I} / \mathrm{R}$ via controlling chemokine levels.

\section{Materials and Methods}

Animals

Male Wistar rats weighing 250-300 g were kept under standard laboratory conditions with free access to food and water. The room temperature was maintained at $37^{\circ} \mathrm{C}$. Animals in each group were housed in the same animal care facility during a 12:12 h light/ dark cycle throughout the study. All experiments were approved by the Ethics Committee of Rafsanjan University of Medical Sciences. All efforts were made to minimize the pain and stress of the rats.

\section{Experimental groups}

The rats were randomly allocated into the following experimental groups (10 rats per group):The sham- operated control group, in which the animals underwent only anesthesia without occlusion. In three other groups, the animals underwent ischemia by 4-VO (4-vessel occlusion) method, then the rats were killed and samples were obtained from brain tissue at $24 \mathrm{~h}$, $48 \mathrm{~h}$ and 1 week after operation. In the postconditioning groups ( 3 groups), all animals underwent 3 cycles of reperfusion/reocclusion and their brain samples were obtained at $24 \mathrm{~h}, 48 \mathrm{~h}$ and 1 week after operation. This model of postconditioning was based on the methods described before.

\section{Animal model and surgical procedure}

The transient global cerebral ischemia was induced by 4-VO method [13]. Rats were first anesthetized intraperitoneally with ketamine hydrochloride $(80 \mathrm{mg} / \mathrm{kg}$ ) and xylazine ( $4 \mathrm{mg} / \mathrm{kg}$ ). On the first day, their common carotid arteries were exposed and both vertebral arteries were electrocauterized permanently. On the next day, cerebral ischemia was induced by occlusion of both common arteries with aneurysm clips for $10 \mathrm{~min}$, then the clips were removed for reperfusion. Rectal temperature was maintained at $37^{\circ} \mathrm{C}$ with a temperature feedback heating pad during the procedure. Rats that had lost their righting reflex, or dilated pupils and were unresponsive to light be used in the experiments $[14,15]$.

\section{Postconditioning protocols}

POCO-45-15/15 animals were subjected to 3 cycles of 15-s/15-s reperfusion/ reocclusion applied after 45 $s$ reperfusion.

\section{Western blot analysis}

Proteins were extracted from freshly dissected and homogenized hippocampus tissues using lysis buffer (Cell Signaling Technology, Beverly, MA, USA).

Samples were used for Sodium Dodecyl Sulfate-PolyAcrylamide Gel Electrophoresis (SDS-PAGE). Immunoblotting and densitometry were performed to quantify the expression of CXCL1, CXCL10 and CXCL12. Equal amounts of protein ( $35 \mathrm{~g}$ ) were loaded and resolved on a $10 \%$ SDS-PAGE and then transferred to nitrocellulose membrane. After blocking with 3\% (w/v) milk in Tween (10 mM Tris, pH 7.4 containing $140 \mathrm{mM} \mathrm{NaCl}, 0.1 \%$ [v/v] Tween 20), the nitrocellulose membrane was incubated overnight at $4^{\circ} \mathrm{C}$ in PBS/Tween containing $3 \%(\mathrm{w} / \mathrm{v})$ milk, including anti-rat CXCL1, CXCL10, CXCL12 and $\beta$-actin monoclonal antibody (Sigma, Mo, USA) (R\&D system, UK). Subsequently, anti-rat horseradish peroxidase- 
conjugated antibodies (Amersham Life Science, UK) (diluted, 1:1000) were used, and to detect localization and amount of protein the enhanced chemiluminescence detection system (Amersham Life Science, UK) was applied. Also $\beta$-actin was used as an internal control to compare the data from different films.

\section{Statistical analysis}

All data were expressed as mean \pm SEM. Comparisons of variables were performed using the Student's t test. The differences were considered significant when $\mathrm{P}<0.05$.

\section{Results}

Results of the present study indicated that the protein levels of both CXCL1 and CXCL10 as members of functionally proinflammatory chemokines decreased in iPOCO treated group compared to untreated groups. In western blotting analysis the expression of chemokines at control level (initiated point of postconditioning) was assigned as $100 \%$ of expression in each chemokine assayed and other groups were proportional to that.

The average concentration of CXCL1 was $98 \%, 98 \%$ and $96.5 \%$ after $24 \mathrm{~h}, 48 \mathrm{~h}$ and 1 week following induction of ischemia and was $94 \%, 78 \%$ and $55 \%$ after $24 \mathrm{~h}$,
$48 \mathrm{~h}$ and 1 week following iPOCO, respectively. These data suggest the regulatory effects of postconditioning on CXCL1 (Figure 1).

Our findings also indicated that the mean concentrations of CXCL10 were 97\%, 98\%, and 96\%, 24 h, 48 h, and 1 week following induction of ischemia, also its mean concentrations were $98 \%, 50 \%$ and $10 \%, 24$ h, 48 $\mathrm{h}$ and 1 week following iPOCO, respectively. The comparison between ischemia group and group received postconditioning suggests the regulatory effect of postconditioning on this chemokine (Figure 2).

We did not detect any alteration in CXCL12 expression and its concentrations did not significantly alter in postconditioning group compared to the control. The mean concentrations of CXCL12 were $98 \%, 94 \%$ and $96 \%$ after $24 \mathrm{~h}, 48 \mathrm{~h}$ and 1 week following induction of ischemia and were $95 \%, 97 \%$ and $96.5 \%$ after 24 h, $48 \mathrm{~h}$ and 1 week following iPOCO, respectively (Figure 3 ). These findings support that postconditioning does not affect this chemokine and is unable to control its expression.

This means that postconditioning is able to control the expression of chemokines CXCL1 and CXCL10 but not the constitutive chemokine CXCL12. On the other

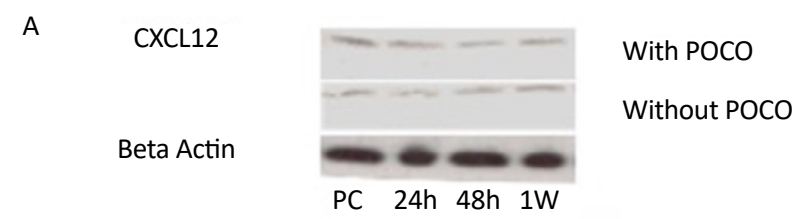

B

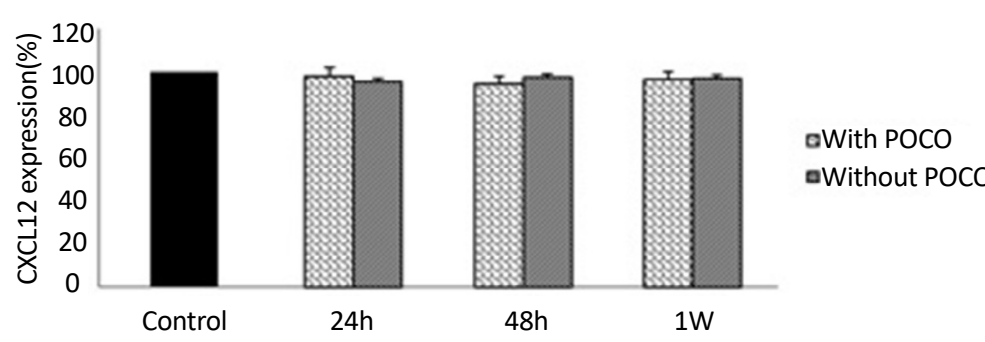

JAMSAT

Figure 1. Expression of CXCL1 at protein level in Wistar rats either with or without POCO

\begin{abstract}
In treatment group, rats were subjected to POCO. At indicated time points, the hippocampus was isolated and homogenized, its protein were extracted and followed by SDS-PAGE and further immunoblotting with specific antibody against CXCL1 and normalized with $\beta$-actin; Profile A: A representative profile of western blotting; Profile B: The expression of CXCL1 at control was assigned as $100 \%$ expression and the expression of CXCL1 at other time points was proportional to that; Data are expressed as mean \pm SEM for 10 separate experiments; * \# Significant difference with the without POCO group.
\end{abstract}


A

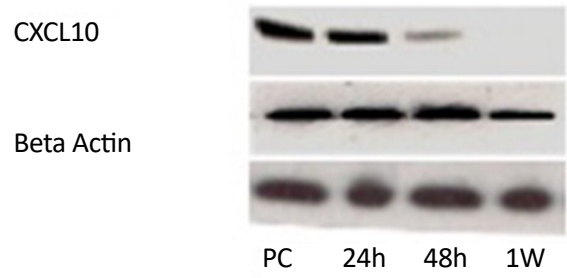

With POCO

Without POCO

B

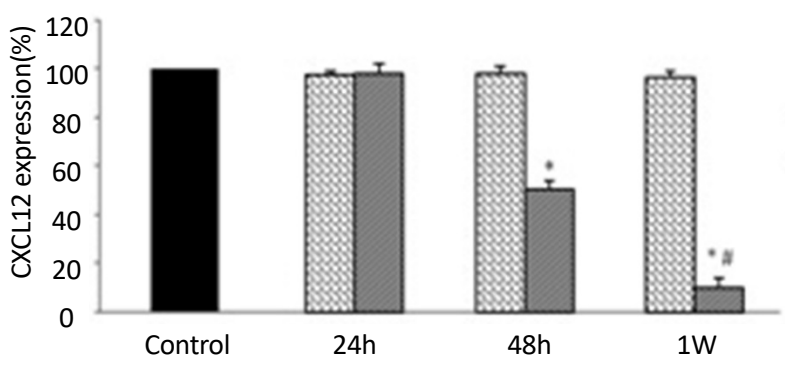

aWith POCO

without POCO

JAMSAT

Figure 2. Expression of CXCL10 at protein level in Wistar rats either with or without POCO

In treatment group, rats were subjected to POCO. At indicated time points, the hippocampus was isolated and homogenized, its proteins were extracted and followed by SDS-PAGE and further immunoblotting with specific antibody against CXCL10 and normalized with $\beta$-actin; Profile A: A representative profile of western blotting; Profile B: The expression of CXCL10 at control was assigned as $100 \%$ expression and CXCL10 expression at other time points was proportional to that. Data are expressed as mean \pm SEM for 10 separate experiments; * Significant difference with control group; * \# Significant difference with the without POCO group.

Abbreviations: PC: Positive Control; W: Week; h: Hour; POCO: Postconditioning

hand, postconditioning reduces inflammation through controlling of proinflammatory CXC chemokines.

\section{Discussion}

The current study aimed to evaluate the expression of CXCL1, CXCL10 and CXCL12 following I/R in the group which received postconditioning protocol.

In the present study, we demonstrated that ischemic postconditioning attenuates brain damage probably via decreased recruitment of immune cells into the brain tissue, decreased expression of the proinflammatory mediators CXC chemokines CXCL1 and CXCL10 but not homeostatic chemokine CXCL12.

These immune cell promigratory proteins have been reported to have potent chemotactic activity for various blood derived cell types, including neutrophils and lymphocytes and therefore play a significant role in the acute inflammatory responses [16]. The decrease in CXCL1 and CXCL10 proteins in brain tissue by postconditioning at the molecular level suggests a mechanism by which inflammation decreases via reduced immune cell effectors recruitment.

In a previous investigation, it has been shown that postconditioning could significantly decrease infarct size and endothelial dysfunction following $\mathrm{I} / \mathrm{R}$ in a canine model [3]. Zhao and colleagues reported that the early phase of reperfusion is a critical phase in injures following ischemia, so treatment strategies in this point may reduce these downstream pathological consequences of $I / R$ injury [3]. The infarct sparing effect of postconditioning in the rabbit [2] was greater than which observed in the rat in the present study but was consistent with that observed in the canine model by Zhao and associates [3].

Oxygen derived free radicals production increases within the first minutes [17] and peaks 4-7 min after the onset of I/R $[18,19]$. This oxidative burst is probably derived in vivo to some extent from activated neutrophils recruited to the lesions [17]. Free oxygen radicals trigger the release of proinflammatory mediators, transcription factors such as NF-kB (NF-kappa B), and stimulate the surface expression of adhesion molecules on coro- 
A

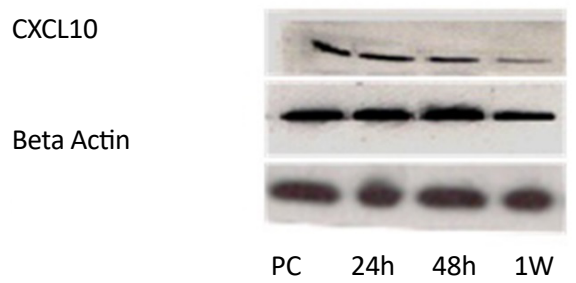

With POCO

Without POCO

\section{B}

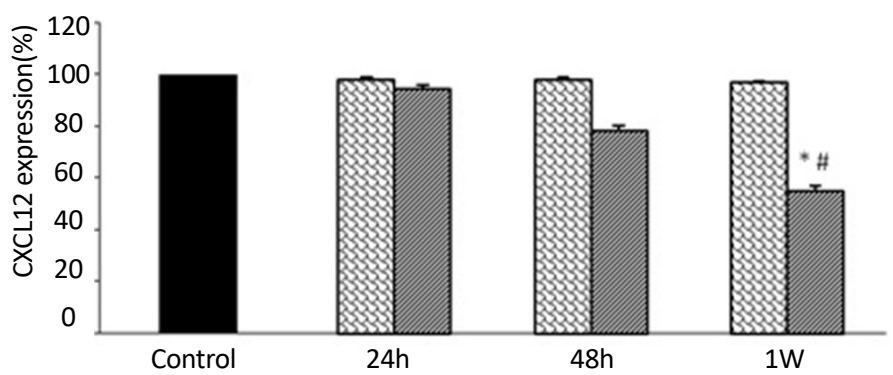

बWith POCO

Without POCO

Figure 3. Expression of CXCL12 at protein level in Wistar rats either with or without POCO

In treatment group, rats were subjected to POCO. At indicated time points, the hippocampus was isolated and homogenized, its proteins were extracted and followed by SDS-PAGE and further immunoblotting with specific antibody against CXCL12 and normalized with $\beta$-actin Profile A: A representative profile of western blotting; Profile B: The expression of CXCL12 at control was assigned as 100\% expression and the expression of CXCL12 at other time points was proportional to that; Data are expressed as mean \pm SEM for 10 separate experiments.

Abbreviations: PC: Positive Control; W: Week; h: Hour; POCO: Postconditioning

nary vascular endothelium [20]. Regarding our previous studies in cell systems, these chemokines are regulated by several cell signaling pathway mediators, including MAPK (Mitogen-activated protein kinases) and NFkB [11, 12 , 21]. Therefore, the higher level of CXCL1 and CXCL10 following I/R could be due to activation of these extra cellular signaling pathways which regulate their expression.

Numerous studies have reported that neutrophils migrate into and accumulate in ischemic-reperfused myocardium [22-24]. Neutrophil accumulation starts immediately at the onset of $\mathrm{I} / \mathrm{R}[23,25,26]$ and continues for $24 \mathrm{~h}$ in canine models [27]. Moreover, it has been showed that a reduction in neutrophil activity and accumulation is associated with a concomitant reduction in infarct size [28-30]. However, it is still controversial whether the presence of neutrophils in reperfused myocardium is related to subsequent injury during reperfusion, or simply an inflammatory response to injury that results from other causes, such as oxygen radicals, cytokines, and so on [31].

I/R injury begins by production of reactive oxygen species, which initially appears to be responsible for the generation of chemotactic activity for neutrophils probably via chemokine expression. It is well-documented that neutrophils and other peripheral blood cell types are an enriched source of chemokines and over expression of CXC chemokines in our model of I/R could also probably be due to the accumulation of neutrophils based on aforementioned evidence.

Furthermore, following $\mathrm{I} / \mathrm{R}$, a spectrum of cytokines and mediators are released which may be responsible for priming neutrophils. These proinflammatory molecules include tumor necrosis factor- $\alpha$ (TNF- $\alpha$ ) and IL- 6 and they can induce direct tissue damage and are also potent activators of neutrophils $[32,33]$. Sequestration of neutrophils and their enzymatic products in lung tissue result in enhancement of microvascular permeability, perivascular and interstitial edema, and pulmonary edema [34]. Cytokine (such as TNF- $\alpha$, IL-6) released along with enzymatic products of neutrophils may also be responsible for up-regulation of proinflammatory CXC chemokines following I/R. The regulating effectors of these mediators on chemokine expression is revealed by this research group $[11,21]$. 
Therefore, inhibiting the production of oxygen radicals, inflammatory cytokine, chemokine releases and neutrophil activation can be therapeutic options for limiting injuries following I/R [35, 36].

Consistent with the findings of previous studies which reported reduction of plasma levels of TNF- $\alpha$ and IL- 6 following both postconditioning and preconditioning, in the current study, the hippocampus protection may be associated with the suppressions of proinflammatory cytokines releases which can attenuate the systemic injury $[37,38]$. In other words, decreased level of proinflammatory cytokines (TNF- $\alpha$, IL-6, etc.) as upstream regulators for expression of CXCL1 and CXCL10 may possibly explain a leading mechanism by which these proinflammatory chemokines decrease following postconditioning in $\mathrm{I} / \mathrm{R}$ in our rat model of postconditioning.

Chemokines, especially CXCL1 and CXCL12 are further demonstrated to play a pivotal role in the regulation of hypoxic preconditioning and pharmacological postconditioning in some cell systems [39-41].

In agreement with our model, human studies indicate that elevated CSF in acute ischemic stroke patients suggests chemokine up-regulation during an early phase of stroke which is in line with studies that showed increased level of CXCL1 and CXCL10 (not CXCL12) in patients with cerebral ischemia. Previous studies also have shown that in vitro cultured astrocytes and microglia [42] as well as neutrophils [43] can respond to cytokines such as TNF- $\alpha$ or interleukin-1 (IL-1), which initiates stroke-induced inflammatory reaction. Thus, both brain resident cells and brain-invaded neutrophils could be potential sources of CXCL1 local production in stroke. This may confirm our findings regarding the elevated level of CXCL1 and CXCL10. This hypothesis is favored by the investigation claiming that mRNA [44] and KC protein [45], homologous to human CXCL1, is elevated in the brain lesion regions of rats subjected to ischemic stroke.

What type of functions in the pathophysiology of stroke may locally release CXCL1 and CXCL10 in our model? CXCL1 and CXCL10 with their potent neutrophils and lymphocytes chemotactic and activating properties could contribute, like other cytokines or chemokines released after cerebral ischemia, to post I/R stroke-derived inflammation involving leucocyte accumulation within ischemic brain. Thus, early local production of both CXCL1 and CXCL10 in I/R may affect the timetable of neutrophils recruitment into ischemic brain following $I / R$, as they initiated their migration within hours to days of I/R with maximal response 24 to $48 \mathrm{~h}$ after I/R [46].
IL-8, another CXC chemokine with potent neutrophils attracting activity, may play the similar role as I/R [47-49].

\section{Conclusion}

The results of present study support the role of chemokines in IPOCO via reduction of inflammation. Also there could be a link between postconditioning, stress, and inflammation through chemokines.

\section{Ethical Considerations}

\section{Compliance with ethical guidelines}

All animal study protocols were approved by the animal care and use committee of the Rafsanjan Medical University.

Funding

The present project was financially supported by Rafsanjan University of Medical Sciences.

\section{Conflict of interest}

The authors declare that there is no conflict of interest

\section{Acknowledgements}

Vice Chancellor of Rafsanjan University of Medical Sciences supported this study.

\section{References}

[1] Koroshetz WJ, Moskowitz MA. Emerging treatments for stroke in humans. Trends in Pharmacological Sciences. 1996; 17(6):227-33. [DOI:10.1016/0165-6147(96)10020-1]

[2] Yang XM, Proctor JB, Cui L, Krieg T, Downey JM, Cohen MV Multiple, brief coronary occlusions during early reperfusion protect rabbit hearts by targeting cell signaling pathways. Journal of the American College of Cardiology. 2004; 44(5):1103-10. [DOI:10.1016/j.jacc.2004.05.060]

[3] Zhao ZQ, Corvera JS, Halkos ME, Kerendi F, Wang NP, Guyton RA et al. Inhibition of myocardial injury by ischemic postconditioning during reperfusion: Comparison with ischemic preconditioning. American Journal of Physiology-Heart and Circulatory Physiology. 2003; 285(2):H579-H88. [DOI:10.1152/ajpheart.01064.2002] [PMID]

[4] Burda J, Danielisová V, Némethová M, Gottlieb M, Matiašová M, Domoráková I, et al. Delayed postconditionig initiates additive mechanism necessary for survival of selectively vulnerable neurons after transient ischemia in rat brain. Cellular and Molecular Neurobiology. 2006; 26(7-8):1141-51. [DOI:10.1007/s10571-0069036-x] [PMID] 
[5] Danielisová V, Némethová M, Gottlieb M, Burda J. The changes in endogenous antioxidant enzyme activity after postconditioning. Cellular and Molecular Neurobiology. 2006; 26(7):1179-89. [DOI:10.1007/s10571-006-9034-z] [PMID]

[6] Zhao H, Sapolsky RM, Steinberg GK. Interrupting reperfusion as a stroke therapy: Ischemic postconditioning reduces infarct size after focal ischemia in rats. Journal of Cerebral Blood Flow \& Metabolism. 2006; 26(9):1114-21. [DOI:10.1038/sj.jcbfm.9600348]

[7] Jiang X, Shi E, Nakajima Y, Sato S. Postconditioning, a series of brief interruptions of early reperfusion, prevents neurologic injury after spinal cord ischemia. Annals of Surgery. 2006; 244(1):14853. [DOI:10.1097/01.sla.0000217608.08582.35]

[8] Rehni AK, Singh N. Role of phosphoinositide 3-kinase in ischemic postconditioning-induced attenuation of cerebral ischemiaevoked behavioral deficits in mice. Pharmacological Reports. 2007; 59(2):192-8. [PMID]

[9] Zhang F, Hu EC, Gerzenshtein J, Lei MP, Lineaweaver WC. The expression of proinflammatory cytokines in the rat muscle flap with ischemia-reperfusion injury. Annals of Plastic Surgery. 2005; 54(3):313-7.

[10] Ahmadi Z, Arababadi MK, Hassanshahi G. CXCL10 activities, biological structure, and source along with its significant role played in pathophysiology of type I diabetes mellitus. Inflammation. 2013; 36(2):364-71. [DOI:10.1007/s10753-012-9555-1] [PMID]

[11] Hassanshahi G, Jafarzadeh A, Ghorashi Z, Sheikholeslami NZ, Dickson AJ. Expression of IP-10 chemokine is regulated by proinflammatory cytokines in cultured hepatocytes. Iranian Journal of Allergy, Asthma and Immunology. 2007; 6(3):115-22.

[12] Asiabanha M, Asadikaram G, Rahnema A, Mahmoodi M, Hasanshahi G, Hashemi M, et al. Chronic opium treatment can differentially induce brain and liver cells apoptosis in diabetic and non-diabetic male and female rats. The Korean Journal of Physiology and Pharmacology. 2011; 15(6):327-32. [DOI:10.4196/ kjpp.2011.15.6.327]

[13] Pulsinelli WA, Brierley JB. A new model of bilateral hemispheric ischemia in the unanesthetized rat. Stroke. 1979; 10(3):267-72 [DOI:10.1161/01.STR.10.3.267]

[14] Wang JY, Shen J, Gao Q, Ye ZG, Yang SY, Liang HW, et al. Ischemic postconditioning protects against global cerebral ischemia/ reperfusion-induced injury in rats. Stroke. 2008; 39(3):983-90. [DOI:10.1161/STROKEAHA.107.499079] [PMID]

[15] Zhang W, Miao Y, Zhou S, Wang B, Luo Q, Qiu Y. Involvement of glutamate transporter-1 in neuroprotection against global brain ischemia-reperfusion injury induced by postconditioning in rats. International Journal of Molecular Sciences. 2010; 11(11):4407. [DOI:10.3390/ijms11114407]

[16] Luangsay S, Wittamer V, Bondue B, De Henau O, Rouger L, Brait $M$, et al. Mouse chemR23 is expressed in dendritic cell subsets and macrophages, and mediates an anti-inflammatory activity of chemerin in a lung disease model. The Journal of Immunology. 2009; 183(10):6489-99. [DOI:10.4049/jimmunol.0901037] [PMID]

[17] Duilio C, Ambrosio G, Kuppusamy P, DiPaula A, Becker LC, Zweier JL. Neutrophils are primary source of O2radicals during reperfusion after prolonged myocardial ischemia. American Journal of Physiology-Heart and Circulatory Physiology. 2001; 280(6):H2649H57. [DOI:10.1152/ajpheart.2001.280.6.H2649] [PMID]

[18] Jeroudi MO, Hartley CJ, Bolli R. Myocardial reperfusion injury: Role of oxygen radicals and potential therapy with anti- oxidants. American Journal of Cardiology. 1994; 73(6):B2-B7. [DOI:10.1016/0002-9149(94)90257-7]

[19] Kevin LG, Camara AKS, Riess ML, Novalija E, Stowe DF. Ischemic preconditioning alters real-time measure of $\mathbf{O 2}$ radicals in intact hearts with ischemia and reperfusion. American Journal of Physiology-Heart and Circulatory Physiology. 2003; 284(2):H566-H74. [DOI:10.1152/ajpheart.00711.2002] [PMID]

[20] Fan H, Sun B, Gu Q, Lafond-Walker A, Cao S, Becker LC. Oxygen radicals trigger activation of NF-kappaB and AP-1 and upregulation of ICAM-1 in reperfused canine heart. American journal of physiology Heart and circulatory physiology. 2002; 282(5):H177886. [DOI:10.1152/ajpheart.00796.2000] [PMID]

[21] Hassanshahi G, Jafarzadeh A, Dickson AJ. Expression of stromal derived factor alpha (SDF-1 $\alpha$ ) by primary hepatocytes following isolation and heat shock stimulation. Iranian Journal of Allergy, Asthma and Immunology. 2008; 7(2):61-8.

[22] Albertine KH, Weyrich AS, Ma XL, Lefer DJ, Becker LC, Lefe AM. Quantification of neutrophil migration following myocardial ischemia and reperfusion in cats and dogs. Journal of Leukocyte Biology. 1994; 55(5):557-66. [DOI:10.1002/jlb.55.5.557]

[23] Dreyer WJ, Michael LH, West MS, Smith CW, Rothlein R, Rossen RD, et al. Neutrophil accumulation in ischemic canine myocardium. Insights into time course, distribution, and mechanism of localization during early reperfusion. Circulation. 1991; 84(1):40011. [DOI:10.1161/01.CIR.84.1.400] [PMID]

[24] Chatelain P, Latour JG, Tran D, de Lorgeril M, Dupras G, Bourassa $M$. Neutrophil accumulation in experimental myocardial infarcts: Relation with extent of injury and effect of reperfusion. Circulation. 1987; 75(5):1083-90. [DOI:10.1161/01.CIR.75.5.1083] [PMID]

[25] Mehta JL, Nichols WW, Mehta P. Neutrophils as potential participants in acute myocardial ischemia: Relevance to reperfusion. Journal of the American College of Cardiology. 1988; 11(6):1309 16. [DOI:10.1016/0735-1097(88)90297-5]

[26] Tsao PS, Aoki N, Lefer DJ, Johnson G, Lefer AM. Time course of endothelial dysfunction and myocardial injury during myocardial ischemia and reperfusion in the cat. Circulation. 1990; 82(4):1402 12. [DOI:10.1161/01.CIR.82.4.1402] [PMID]

[27] Zhao ZQ, Nakamura M, Wang NP, Velez DA, Hewan-Lowe KO, Guyton RA, et al. Dynamic progression of contractile and endothelial dysfunction and infarct extension in the late phase of reperfusion. Journal of Surgical Research. 2000; 94(2):133-44. [DOI:10.1006/jsre.2000.6029] [PMID]

[28] Curtis WE, Gillinov AM, Wilson IC, Bator JM, Burch RM, Cameron $\mathrm{DE}$, et al. Inhibition of neutrophil adhesion reduces myocardial infarct size. The Annals of Thoracic Surgery. 1993; 56(5):1069-73. [DOI:10.1016/0003-4975(95)90015-2]

[29] Forman MB, Virmani R, Puett DW. Mechanisms and therapy of myocardial reperfusion injury. Circulation. 1990; 81(3 Suppl):IV69-78. [PMID]

[30] Hartman JC, Anderson DC, Wiltse AL, Lane CL, Rosenbloom $\mathrm{CL}$, Manning $\mathrm{AM}$, et al. Protection of ischemic/reperfused canine myocardium by $\mathrm{CL18} / 6$, a monoclonal antibody to adhesion molecule ICAM-1. Cardiovascular Research. 1995; 30(1):47-54 [DOI:10.1016/0008-6363(95)00015-1]

[31] Baxter GF. The neutrophil as a mediator of myocardial ischemiareperfusion injury: Time to move on. Basic Research in Cardiology. 2002; 97(4):268-75. [DOI:10.1007/s00395-002-0366-7] [PMID] 
[32] Bergin PF, Doppelt JD, Kephart CJ, Benke MT, Graeter JH, Holmes AS, et al. Comparison of minimally invasive direct anterior versus posterior total hip arthroplasty based on inflammation and muscle damage markers. The Journal of Bone and Joint Surgery-American Volume. 2011; 93(15):1392-8. [DOI:10.2106/jbjs.j.00557]

[33] Tüzün E, Li J, Wanasen N, Soong L, Christadoss P. Immunization of mice with $T$ cell-dependent antigens promotes IL- 6 and TNF- $\alpha$ production in muscle cells. Cytokine. 2006; 35(1-2):100-6. [DOI:10.1016/j.cyto.2006.05.009]

[34] Park JW, Ma SN, Song ES, Song CH, Chae MR, Park BH, et al. Pulmonary damage by Vibrio vulnificus cytolysin. Infection and Immunity. 1996; 64(7):2873-6. [PMID] [PMCID]

[35] Fan C, Zwacka RM, Engelhardt JF. Therapeutic approaches for is chemia/reperfusion injury in the liver. Journal of Molecular Medicine. 1999; 77(8):577-92. [DOI:10.1007/s001099900029] [PMID]

[36] Tsung A, Kaizu T, Nakao A, Shao L, Bucher B, Fink MP, et al. Ethy pyruvate ameliorates liver ischemia-reperfusion injury by decreasing hepatic necrosis and apoptosis. Transplantation. 2005; 79(2):196-204. [DOI:10.1097/01.tp.0000151681.07474.2e]

[37] Packard AE, Leung PY, Vartanian KB, Stevens SL, Bahjat FR, Stenzel-Poore MP. TLR9 bone marrow chimeric mice define a role for cerebral TNF in neuroprotection induced by $\mathrm{CpG}$ preconditioning Journal of Cerebral Blood Flow \& Metabolism. 2012; 32(12):2193200. [DOI:10.1038/jcbfm.2012.140]

[38] Sakata H, Narasimhan P, Niizuma K, Maier CM, Wakai T, Chan $\mathrm{PH}$. Interleukin 6-preconditioned neural stem cells reduce ischaemic injury in stroke mice. Brain. 2012; 135(11):3298-310. [DOI:10.1093/brain/aws259] [PMID] [PMCID]

[39] Masayuki K, Tao Sheng L, Takahiro K, Mako O, Shu Lan Q, Kimikazu $H$. Increased expression of CXCR4 and integrin $\alpha M$ in hypoxia-preconditioned cells contributes to improved cell retention and angiogenic potency. Journal of Cellular Physiology. 2009; 220(2):508-14. [DOI:10.1002/jcp.21803] [PMID]

[40] Tang YL, Zhu W, Cheng M, Chen L, Zhang J, Sun T, et al. Hypoxic preconditioning enhances the benefit of cardiac progenitor cell therapy for treatment of myocardial infarction by inducing CXCR4 expression. Circulation Research. 2009; 104(10):1209-16. [DOI:10.1161/CIRCRESAHA.109.197723] [PMID] [PMCID]

[41] Voigtsberger MDS, Lachmann MDPDRobert A, Leutert MDAnik C, Schläpfer MDM, Booy C, Reyes L, et al. Sevoflurane ameliorates gas exchange and attenuates lung damage in experimenta lipopolysaccharide-induced lung injury. Anesthesiology. 2009; 111(6):1238-48. [DOI:10.1097/ALN.0b013e3181bdf857] [PMID]

[42] Janabi N, Hau I, Tardieu M. Negative feedback between prostaglandin and $\alpha$ - and $\beta$-Chemokine synthesis in human microglial cells and astrocytes. The Journal of Immunology. 1999; 162(3):1701-6. [PMID]

[43] Patrizia S, Carlo L, Cristina P, Paola A, Alberto M, Silvano S, et al. Neutrophils produce biologically active macrophage inflammatory protein-3 $\alpha$ (MIP-3 $\alpha$ ) / CCL20 and MIP-3 $\beta$ / CCL19. European Journal of Immunology. 2001; 31(7):1981-8. [DOI:10.1002/15214141(200107)31:73.0.CO; 2-X]

[44] Liu T, Young PR, McDonnell PC, White RF, Barone FC, Feuerstein GZ. Cytokine-induced neutrophil chemoattractant mRNA expressed in cerebral ischemia. Neuroscience Letters. 1993; 164(12):125-8. [DOI:10.1016/0304-3940(93)90873-j]

[45] Yamasaki Y, Matsuo $Y$, Matsuura N, Onodera H, Itoyama $Y$, Kogure $\mathrm{K}$. Transient increase of cytokine-induced neutrophil che- moattractant, a member of the interleukin-8 family, in ischemic brain areas after focal ischemia in rats. Stroke. 1995; 26(2):318-23. [DOI:10.1161/01.STR.26.2.318] [PMID]

[46] Becker KJ. Inflammation and acute stroke. Current Opinion in Neurology. 1998; 11(1):45-9. [DOI:10.1097/00019052-19980200000008] [PMID]

[47] Kostulas N, Kivisäkk P, Huang Y, Matusevicius D, Kostulas V, Link $\mathrm{H}$. Ischemic stroke is associated with a systemic increase of blood mononuclear cells expressing interleukin-8 mRNA. Stroke. 1998; 29(2):462-6. [DOI:10.1161/01.STR.29.2.462] [PMID]

[48] Kostulas N, Pelidou SH, Kivisäkk P, Kostulas V, Link H. Increased IL-1 $\beta$, IL-8, and IL-17 mRNA expression in blood mononuclear cells observed in a prospective ischemic stroke study. Stroke. 1999; 30(10):2174-9. [DOI:10.1161/01.STR.30.10.2174] [PMID]

[49] Tarkowski E, Rosengren L, Blomstrand C, Wikkelsö C, Jensen C Ekholm S, et al. Intrathecal release of pro-and anti-inflammatory cytokines during stroke. Clinical \& Experimental Immunology. 1997; 110(3):492-9. [DOI:10.1046/j.1365-2249.1997.4621483.x] [PMCID] 